\title{
Towards a Universal Methodology for Performance Evaluation of Electrical Impedance Tomography Systems Using Full Reference SNR
}

\author{
Yu Wu, Dai Jiang, Nazanin Neshatvar, Farnaz Fahimi Hanzaee, and Andreas Demosthenous \\ Department of Electronic and Electrical Engineering, University College London, Torrington Place, London WC1E 7JE, UK \\ e-mail: yu.wu.09@ucl.ac.uk; a.demosthenous@ucl.ac.uk
}

\begin{abstract}
This paper describes a simple and reproducible methodology towards a universal figure-of-merit (FoM) for evaluating the performance of electrical impedance tomography (EIT) systems using reconstructed images. Based on objective fullreferencing and signal-to-noise ratio, the method provides a visually distinguishable hot-map and two new FoM factors, to address the issues where common electrical parameters are not directly related to the quality of EIT images. The paper describes the method with simulation results and develops a 16 electrode EIT system using an ASIC front-end for evaluation using the proposed method. The measured results show both visually and in terms of the proposed FoM factors, the impact on recorded EIT images with different current injection amplitudes.
\end{abstract}

Keywords - Electrical impedance tomography (EIT), system performance evaluation, figure-of-merit (FoM)

\section{INTRODUCTION}

Electrical impedance tomography (EIT) is an impedance measurement technique that uses the tomography principle to reconstruct an image which illustrates the inner impedance distribution of the subject under test (SUT). This technique has been widely used in many biomedical applications, such as stroke [1], cancer detection [2], lung function monitoring [3], [4], and recently for monitoring the activity of the vagus nerve during stimulation [5].

Given the potential of EIT, a large number of EIT systems have been developed. At the time of writing, from 2015 to 2019, by searching 'Electrical impedance tomography system, and EIT system' resulted in 835 papers in IEEEXplore alone. However, there are only a handful of papers that describe an evaluation method for EIT systems [6]-[8], and a universally adopted EIT figure-of-merit (FoM) is far from being recognized. To understand the problem, it is important to first know the operation of an EIT system which is illustrated in Fig. 1(a). An electrical system must measure the data from the SUT, and then using reconstruction software, the data is converted into an image by solving a highly ill-posed inverse problem. Therefore, to evaluate the performance of the electrical systems, one must: (A) use identical SUT and reconstruction software; (B) as an imaging system, the comparison should be ultimately demonstrated in terms of an image quality factor; and (C) in order to be widely adopted, the method should be simple and reproducible.

Regarding point (A), most of the systems have been tested on their own SUT (typically a phantom using a water tank); an example is shown in Fig. 1(b) [9]-[12]. With phantom

This work is funded by the European Commission under H2020EU.1.2.2. - FET Proactive (Agreement ID: 824071).

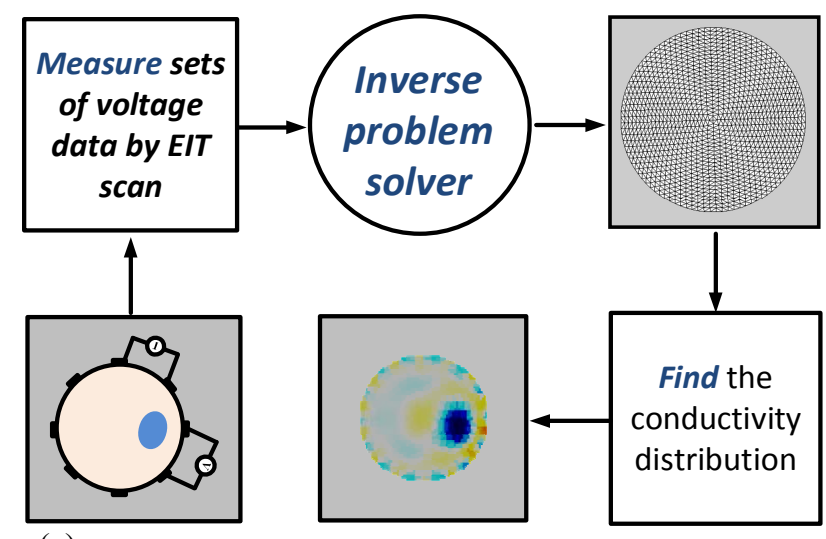

(a)
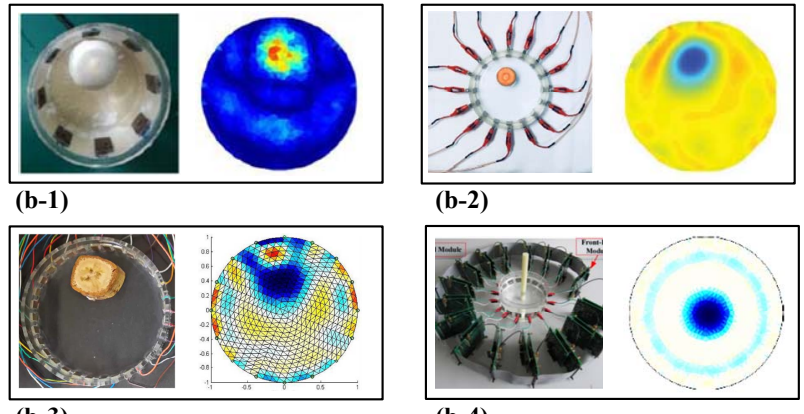

(b-2)

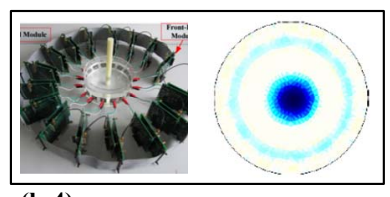

(b-4)

Fig. 1. (a) Overview of EIT operation. (b-1) $9.5 \mathrm{~mm}$ (diameter) tank with raw egg in tap-water [9], (b-2) $125 \mathrm{~mm}$ tank with carrot in $0.1 \% \mathrm{w} / \mathrm{v}$ salt solution [10], (b-3) $110 \mathrm{~mm}$ tank with banana in 'normal' saline solution [11], (b-4) $200 \mathrm{~mm}$ tank with nylon bar in tap water with conductivity of $347 \mu \mathrm{S} / \mathrm{cm}[12]$.

dimensions, electrode size, concentration of the saline solution and type, and position of object inserted - all being different, comparing one system to another by virtue of image results is almost impossible. As for the reconstruction software, fortunately, EIDORS [13] has been dominantly used in the EIT community. This reconstruction software offers three main algorithms, namely the back-projection, GREIT and GaussNewton.

Regarding point (B), even though electrical design comparison for EIT systems was studied in [7], [8], a quantitatively analyzed relationship between measurement data (which could reflect the hardware performance) and image results, is yet to be established due to the nature of solving an 
ill-posed inverse problem. Without this relationship, justification of electrical specifications or functions is difficult.

Regarding point (C), an attempt to evaluate the system performance through image quality has been studied in [6]. However, with over ten quality parameters to define, in addition to a phantom with a robotic-arm required to reproduce the test, the approach in [6] is unlikely to be widely adopted. As EIT fundamentally does not yet offer particularly high resolution, the current practice of placing the water tank beside its EIT image [Fig. 1(b)] for visual assessment is not ideal.

In this paper, a simple and reproducible methodology for evaluating the performance of EIT systems is presented. The method establishes a direct relationship between captured data and image quality based on color mapping of signal-to-noise ratio (SNR) onto an image for easy recognition. An example 16-electrode EIT system has been developed and evaluated using the proposed method.

The rest of the paper is organized as follows. Section II presents the methodology with simulation results analyzed. Section III presents the design and implementation of the 16 electrode EIT system for evaluation. Section IV describes the results with conclusions in Section V.

\section{Methodology Model Simulation}

Since it is not possible to carry out EIT image quality assessment subjectively due to its low resolution, an objective full-reference (FR) method is proposed. FR aims to compare the color accuracy of the test image to a reference image which is considered as the ground truth. Thus, first an ideal reference image must be generated.
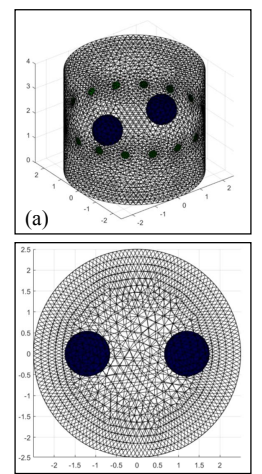

(b)

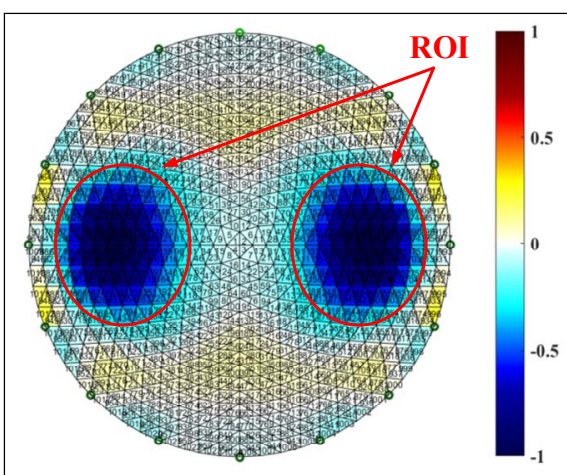

(c)
Fig. 2. (a) 3D forward model. (b) Top-view of the 3D model. (c) EIT image generated using the simulation data. The color-bar indicates normalized conductance and is applied throughout this paper.

\section{A. Reference Image Generation}

In this paper, a 16-electrode EIT system has been chosen as an example. For model simulation, the generation of images is based on EIDORS v3.9.1 forward problem solving [14]. The forward model is built as shown in Fig. 2(a). It comprises two spheres inside a homogenous tank model. Two insulated spheres are inserted at the centre plane of the tank to cause an inhomogeneous conductive re-distribution. By placing the
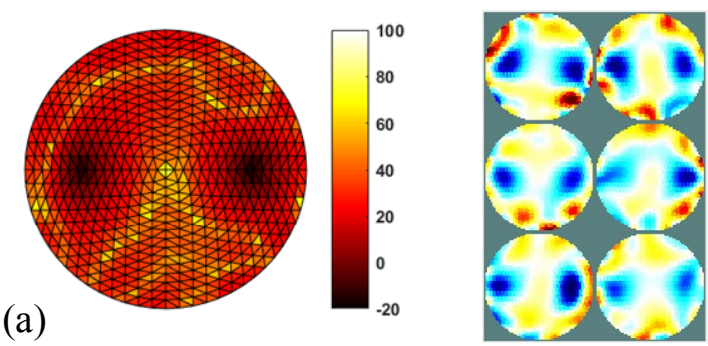

(b)
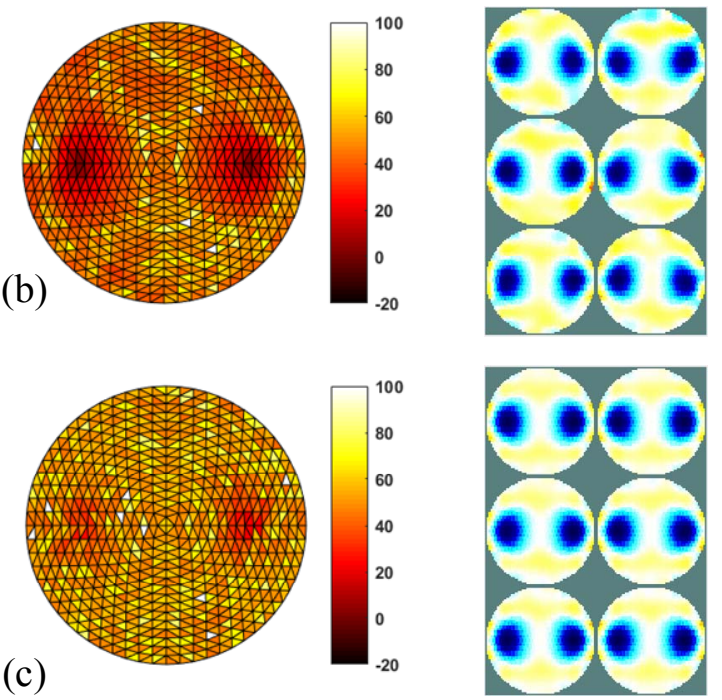

Fig. 3. FR-SNR mapping (hot-maps) together with the corresponding EIT images using simulation data (a) $\pm 5 \%$ variation noise, (b) $\pm 1.25 \%$ variation noise, (c) $\pm 0.25 \%$ variation noise. The color-bar indicates FR-SNR.

electrodes at the centre plane of the tank and solving the forward problem, the ideal homogeneous and inhomogeneous datasets can be generated for EIT differential imaging.

Solving the inverse problem using the Gauss-Newton algorithm, an image [Fig. 2(c)] can be reconstructed using the simulated data. This image is considered the 'ideal' reference using the EIT technique to represent the inner impedance crosssection distribution [Fig. 2(b)] that the electrode array surrounds.

\section{B. Full-Reference Evalution Using SNR}

In many EIT system papers [4], [15]-[17], SNR is the most frequently used parameter to evaluate system performance. It is defined as $S N R_{i}=10 \log _{10}\left(\left[\bar{V}_{i}\right]^{2} / \operatorname{VAR}_{\left[V_{i}\right]}\right)$ from a multiple measurement dataset, where $i$ is the channel number, $\bar{V}$ is the mean, and VAR is the variance of the dataset. However, this SNR only reflects variations between multiple measurements in each channel; it does not reflect how well the data collected is able to reconstruct an image.

From Fig. 2(c), it can be seen that the EIT image comprises 1024 pixels. After solving the inverse problem, each pixel has a color code assigned according to the EIDORS color mapping. Any difference in the color code between the test image and the reference image is considered an error. We define the FR-SNR as:

$$
\mathrm{FR}-\mathrm{SNR}_{i}=20 \log _{10} \frac{\text { Cref }_{i}}{\mid \text { Cref }_{i}-\text { Ctest }_{i} \mid}
$$


where $i$ is the pixel number, and Cref and Ctest are color codes for reference and test image respectively, when $\operatorname{Cref}_{i}=$ Ctest $_{i}$; SNR is set to $100 \mathrm{~dB}$ to avoid an infinite result. By replacing the original EIT image color code to the FR-SNR for each pixel, a hot- map can be reconstructed showing the image quality.

Using the simulated data, a percentage variation is randomly added as noise to the inhomogeneous dataset and the effect of these variations on the data can be illustrated by an image as shown in Fig. 3. For a 16-electrode system, there are 208 measurements in the dataset for one frame of image. Up to the chosen percentage value, each measurement is multiplied by a random percentage variation. In Fig. 3, three percentages are applied; $\pm 5 \%, \pm 1.25 \%$ and $\pm 0.25 \%$. 100 frames of datasets for each percentage are generated and the FR-SNR are calculated for each pixel in each image. The pixels' FR-SNR are then averaged and used to plot the hot-map. Next to each hot-map, 6 EIT images are shown to demonstrate the appearance of the actual EIT images with these percentage variations applied. Over the whole image region, the FR-SNR on average are 30 $\mathrm{dB}, 45 \mathrm{~dB}$ and $54 \mathrm{~dB}$ for $\pm 5 \%, \pm 1.25 \%$ and $\pm 0.25 \%$, respectively. In the region of interest (ROI), which is defined by the spheres' area as in the reference image Fig. 1(c), the ROI FR-SNR on average are $12 \mathrm{~dB}, 28 \mathrm{~dB}$ and $47 \mathrm{~dB}$ for $\pm 5 \%$, $\pm 1.25 \%$ and $\pm 0.25 \%$, respectively.

\section{Reference Image for System Evalution}

For system evaluation, a 32-electrode resistive phantom is used instead of a water tank. This phantom [18] shown in Fig. 4 is easy to implement and commercially available.

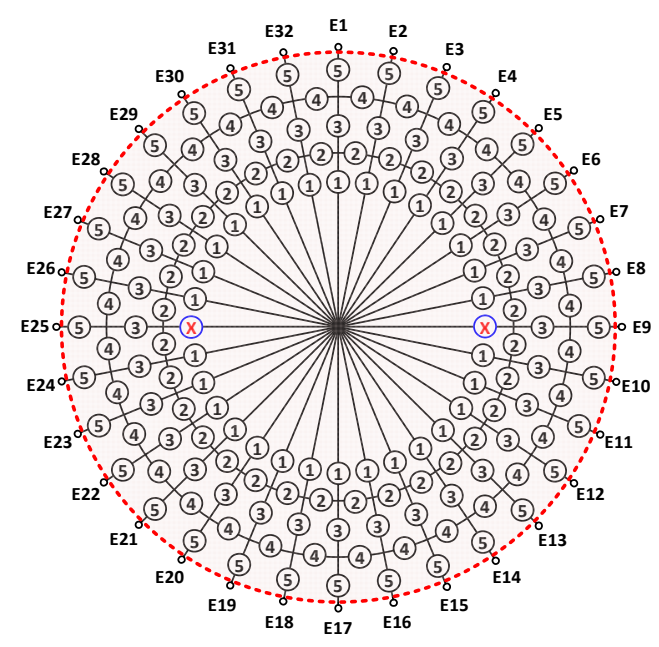

Fig. 4. EIT resistive phantom [18] used for evaluation. Resistive elements (5) $=100 \Omega$, (4) $=196 \Omega$, (3) $=63.4 \Omega$, (2) $=6.34 \Omega$, (1) $=68.1 \Omega$.

By skipping electrodes, the phantom can be used for 8,16 , or 32 electrode systems. The ideal dataset can be generated using most of the electrical simulation tools. The two $\mathrm{X}$ resistive elements can be toggled between $68.1 \Omega$ and $0 \Omega$ for homogeneous and inhomogeneous datasets for EIT differential imaging.
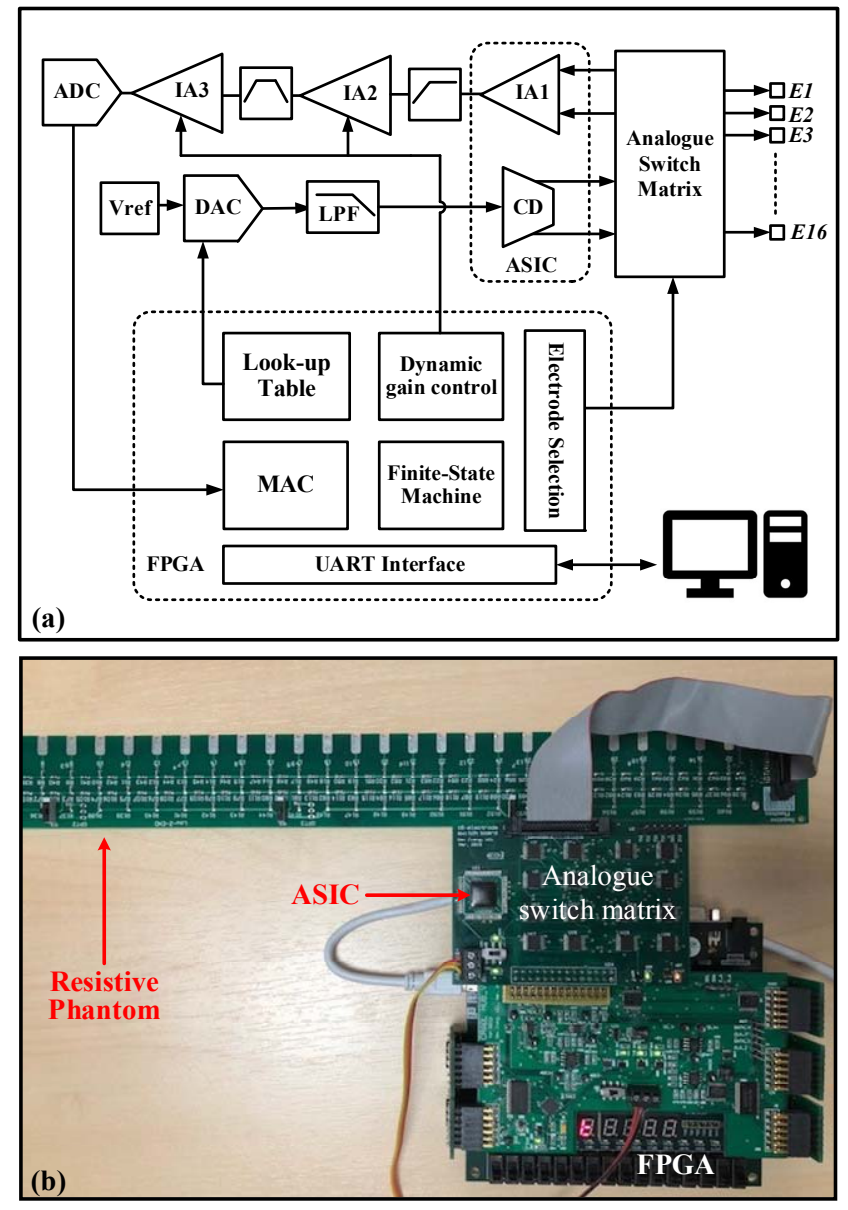

Fig. 5. (a) System architecture of a 16-electrode EIT system. (b) System test set-up.

\section{EIT SYSTEM IMPLEMENTATION}

A 16-electrode wideband EIT system was implemented to evaluate the FR method. Fig. 5(a) shows the architecture of the system. The scan cycles for current drive and voltage recording are facilitated by an analog switch matrix, which selectively connects the 16 electrodes to either the positive or negative output of the fully differential current driver (CD), or either the positive or negative input of the first stage instrumentation amplifier (IA1). The CD and IA1 are integrated in a high performance application specific integrated circuit (ASIC) in $0.35 \mu \mathrm{m}$ CMOS technology [15].

The CD can generate fully-differential ac sinewave currents for frequencies up to $1 \mathrm{MHz}$ with a maximum peak-to-peak amplitude of $6 \mathrm{~mA}$. For EIT measurement, the $\mathrm{CD}$ is driven by a sinusoidal signal generated from a 12-bit digital-to-analog converter (DAC), where the input to the DAC is from the lookup table inside a Xilinx Artix-7 FPGA. The frequency and amplitude of the DAC output can be programmed to vary the drive current for EIT measurement. The voltage on the selected electrode pairs in an EIT scan is amplified in three stages. The first stage integrated IA1 has a fixed gain of 10 . The gains of the second and third stage amplifier, IA2 and IA3, are dynamically 
adjusted by the FPGA during an EIT scan, depending on the relative distance between the voltage recording electrode pair and the current drive electrode pair. The total gain of the 3-stage amplification is adjustable between 10 and $2,000 \mathrm{~V} / \mathrm{V}$. The amplified voltage is digitized by a 12-bit analog-to-digital converter (ADC), and then processed in a multiplieraccumulator (MAC) in the FPGA to derive, using coherence detection, the real and imaginary values of the measured impedance. The results are sent to a computer via a UART interface for image reconstruction. A finite-state machine in the FPGA controls the operation of the system.

\section{System Evaluation Using FR-SNR}

\section{A. Reference Image Generation}

The dataset for the ideal reference image for use in the FR comparison is generated through simulation using Cadence with adjacent scan pattern. Benefiting from differential imaging, and the fact that the phantom uses pure resistive elements, for simulation data, the amplitude and frequency of current drive has no effect on the end image result. A physical phantom is also designed on a PCB using $1 \%$ tolerance resistors to minimize the error between simulation data and measured data. Resistor tolerance can be neglected, provided a difference in FR-SNR can be demonstrated when bench-marking other controlled variables e.g. errors which are mostly contributed by the EIT system itself.

Note that simply computing errors between the simulated data and measured data can provide an accuracy parameter as defined in [6]. However, it still does not give an image related FoM, and as shown in Section II-B, data accuracy is not linearly related to the quality of reconstructed EIT image, thus the effectiveness of this method is limited.

\section{B. System Performance Evaluation}

The system was tested on the phantom using adjacent scan pattern. The test equipment is shown in Fig. 5(b). The drive current amplitude was set to $4 \mathrm{~mA}_{\mathrm{p}-\mathrm{p}}$ with a frequency of 125 $\mathrm{kHz}$. 50 frames of dataset were captured and the average system FR-SNR is shown in Fig. 6(a). With the other settings remaining unchanged, the same procedure was repeated with the drive current amplitude reduced to $1.4 \mathrm{~mA}_{\mathrm{p}-\mathrm{p}}$; the results are shown in Fig. 6(b).

Within each box in Fig. 6, on the right side, the top image is the ideal reference, and the bottom image is one of the 50 images measured using the system for comparison. For both settings, the measured EIT images are very similar to the eye, while image error and the difference can be easily identified on the FRSNR hot-maps. The overall regional FR-SNR is $40 \mathrm{~dB}$ for $4 \mathrm{~mA}_{\mathrm{p}-\mathrm{p}}$ and is reduced to $32.3 \mathrm{~dB}$ for $1.4 \mathrm{~mA}_{\mathrm{p}-\mathrm{p}}$. The ROI FR-SNR are $31.1 \mathrm{~dB}$ and $21.4 \mathrm{~dB}$ for 4 and $1.4 \mathrm{~mA}_{\mathrm{p}-\mathrm{p}}$ respectively. Knowing the benefit that a higher current drive could improve the EIT image an appropriate decision could be made about the system specification according to the application.
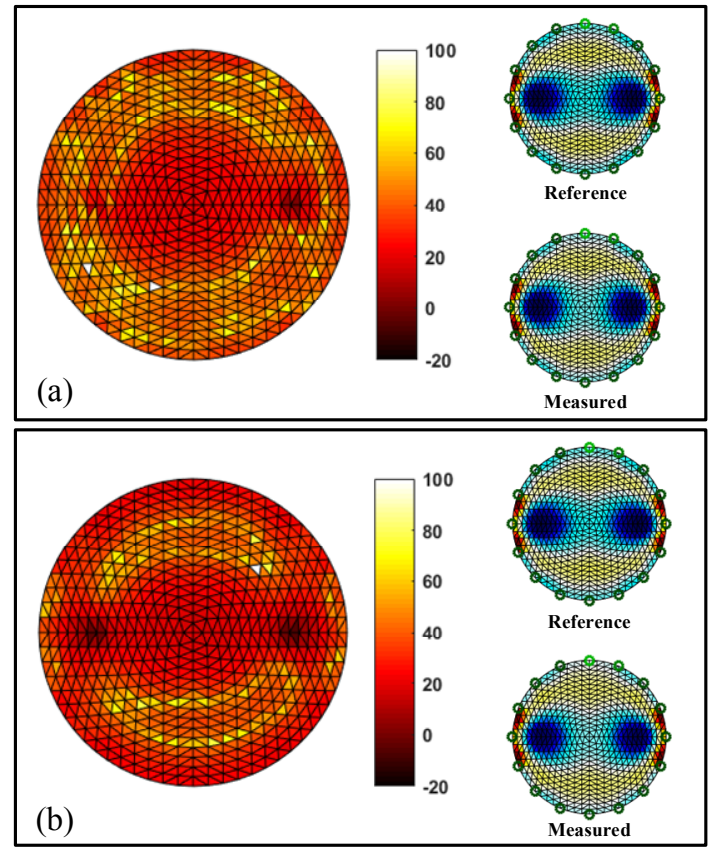

Fig. 6. FR-SNR mapping using measured data from the 16 electrode EIT system developed. On the left hot maps, on the right, the top image is the ideal reference, and the bottom is one of the system measured images for comparison using (a) $4 \mathrm{~mA}_{\mathrm{p}-\mathrm{p}}$ current amplitude, and (b) $1.4 \mathrm{~mA}_{\mathrm{p}-\mathrm{p}}$ current amplitude

\section{CONCLUSION}

A simple and reproducible method has been proposed towards a universal FoM for evaluating the performance of EIT systems. Based on objective full-referencing and combined with SNR, the method addresses the issue that common electrical parameters are not easily directly related to the quality of EIT images, making it difficult to quantify specifications. By providing a visually distinguishable hot-map and two FoM factors (regional and ROI FR-SNR), the evaluation and benchmarking of EIT systems are made simpler and more straightforward.

\section{REFERENCES}

[1] A. Romsauerova, A. McEwan, L. Horesh, R. Yerworth, R. H. Bayford, and D. S. Holder, "Multi-frequency electrical impedance tomography (EIT) of the adult human head: Initial findings in brain tumours, arteriovenous malformations and chronic stroke, development of an analysis method and calibration," Physiol. Meas., vol. 5, no. 5, 2006.

[2] S. Hong et al., "A $4.9 \mathrm{~m} \Omega$-sensitivity mobile electrical impedance tomography IC for early breast-cancer detection system," IEEE J. SolidState Circuits, vol. 50, no. 1, pp. 245-257, 2015.

[3] Y. Wu, D. Jiang, A. Bardill, S. De Gelidi, R. Bayford, and A. Demosthenous, "A high frame rate wearable EIT system using active electrode ASICs for lung respiration and heart rate monitoring," IEEE Trans. Circuits Syst. I Regul. Pap., vol. 65, no. 11, pp. 3810-3820, 2018.

[4] B. Liu et al., "A 13-channel 1.53-mW 11.28-mm2 electrical impedance tomography $\mathrm{SoC}$ based on frequency division multiplexing for lung physiological imaging," IEEE Trans. Biomed. Circuits Syst., 2019, doi: 10.1109/TBCAS.2019.2927132.

[5] C. A. R. Chapman et al., "Electrode fabrication and interface optimization for imaging of evoked peripheral nervous system activity with electrical impedance tomography (EIT)," J. Neural Eng., vol. 16, no. 1, 2019.

[6] M. Yasin, S. Böhm, P. O. Gaggero, and A. Adler, "Evaluation of EIT 
system performance," Physiol. Meas., vol. 32, no. 7, 2011.

[7] M. Takhti and K. Odame, "Structured design methodology to achieve a high SNR electrical impedance tomography," IEEE Trans. Biomed. Circuits Syst., vol. 13, no. 2, pp. 364-375, 2019.

[8] A. McEwan, G. Cusick, and D. S. Holder, "A review of errors in multifrequency EIT instrumentation," Physiol. Meas., vol. 28, no. 7, 2007.

[9] Z. Xu et al., "Development of a portable electrical impedance tomography system for biomedical applications," IEEE Sens. J., vol. 37, no. $6,2018$.

[10] C. Tan, S. Liu, J. Jia, and F. Dong, "A wideband electrical impedance tomography system based on sensitive bioimpedance spectrum bandwidth," IEEE Trans. Instrum. Meas., vol. 69, no. 1, pp. 144-154 2020.

[11] M. S. Tšoeu and M. R. Inggs, "Fully parallel electrical impedance tomography using code division multiplexing," IEEE Trans. Biomed. Circuits Syst., vol. 10, no. 3, pp. 556-566, 2016.

[12] L. Miao et al., "A new symmetric semi-parallel electrical impedance tomography (EIT) system - I: The design,” in Proc. IST 2016 - 2016 IEEE Int. Conf. Imaging Systems and Techniques, 2016.

[13] EIDORS, "EIDORS: Electrical Impedance Tomography and Diffuse Optical Tomography Reconstruction Software." [Online]. Available: http://eidors3d.sourceforge.net/. [Accessed: 03-Oct-2019].
[14] EIDORS, "EIDORS: Solving a 3D forward model with EIDORS." [Online].

Available: http://eidors3d.sourceforge.net/tutorial/EIDORS basics/forward solver s 3d.shtml. [Accessed: 10-Oct-2019].

[15] Y. Wu, D. Jiang, A. Bardill, R. Bayford, and A. Demosthenous, "A 122 fps, $1 \mathrm{MHz}$ bandwidth multi-frequency wearable EIT belt featuring novel active electrode architecture for neonatal thorax vital sign monitoring," IEEE Trans. Biomed. Circuits Syst., vol. 13, no. 5, pp. 927 937, 2019.

[16] S. Hong, J. Lee, J. Bae, and H. J. Yoo, “A $10.4 \mathrm{~mW}$ electrical impedance tomography SoC for portable real-time lung ventilation monitoring system," IEEE J. Solid-State Circuits, vol. 50, no. 11, pp. 2501-2512, 2015.

[17] S. Khan, P. Manwaring, A. Borsic, and R. Halter, "FPGA-based voltage and current dual drive system for high frame rate electrical impedance tomography," IEEE Trans. Med. Imaging, vol. 34, no. 4, pp. 888-901, 2015.

[18] Swisstom, "Swisstom BB2 Product Information," Rev.004, 2013. [Online]. Available: http://www.swisstom.com/en/products/swisstombb2_en. [Accessed: 10-Oct-2019]. 